\title{
Embedded intelligent vacuum monitoring method of high voltage circuit breaker based on Internet of things
}

\author{
LIU Wei-xue \\ \{tiantiankaixin255@tom.com\} \\ (Collage of Mathematics and Compute Science,Chifeng University,Chifeng 024000,china)
}

\begin{abstract}
A certain degree of vacuum is the premise to ensure that the intelligent high-voltage circuit breaker will extinguish the arc during operation, and the degree of vacuum will drop in operation due to some reasons, so it needs to be monitored frequently. In view of the low accuracy of the traditional embedded intelligent high-voltage circuit breaker vacuum monitoring method, the embedded intelligent high-voltage circuit breaker vacuum monitoring method based on the Internet of things is proposed Law. Based on the analysis of the principle of embedded intelligent high-voltage circuit breaker vacuum degree monitoring, using the Internet of things sensor technology, the embedded intelligent high-voltage circuit breaker vacuum degree monitoring. The experimental results show that the proposed method has high accuracy and good monitoring effect, which is of great significance to the construction of smart grid.
\end{abstract}

Keywords: Internet of things technology;Embedded system;Intelligent high voltage circuit breaker;Vacuum degree;

\section{Introduction}

The high-voltage circuit breaker has dual functions of control and protection in the power system. It is one of the equipment with the largest maintenance workload in the power system equipment. Its overall operation, maintenance and repair are closely related to the whole power system. Its reliable breaking capacity is indispensable for the construction of a safe and reliable smart grid, and plays an important role in the power system [1]. In recent years, because of its unique advantages, vacuum circuit breaker has been gradually applied in distribution network at home and abroad. In Baoding area, more than $90 \%$ of the circuit breakers used in $10 \mathrm{kV}$ distribution network are vacuum circuit breakers, and vacuum circuit breakers are used in new and reconstructed distribution network projects. The main performance index of vacuum circuit breaker is the vacuum degree in the arc extinguishing chamber. The vacuum value directly affects the arc extinguishing effect, and then affects the breaking capacity of the switch [2]. If the vacuum degree changes to a certain critical value, the normal arc extinguishing function cannot be completed, and the load current cannot be cut off, which will directly damage the protected lines and equipment; it will also cause power grid accidents, such as large-area power outage, affect 
people's normal life order, and cause great economic losses. The on-line monitoring system of vacuum degree of high-voltage circuit breaker can know the working state and defect position of circuit breaker in time, reduce the premature or unnecessary power failure test and maintenance, reduce the maintenance workload, reduce the maintenance cost, improve the pertinence of maintenance, and significantly improve the reliability of power system. Therefore, through the on-line monitoring of the vacuum degree parameters of the embedded intelligent high-voltage circuit breaker, further reasonable fault analysis can provide practical guidance for the safe, reliable and economic operation of the power system [3-4].

At present, domestic and foreign research in this area has made some achievements. In reference [5], a vacuum degree detection system based on panning discharge principle is proposed, and a complete on-line vacuum degree detection system of high-voltage vacuum circuit breaker is designed. The system is mainly composed of power supply system and signal acquisition and processing system, which realizes the power supply of vacuum sensor and other equipment. Under the environment of high-voltage and strong electromagnetic field, it can measure the vacuum degree signal with high precision and directly detect the vacuum degree of vacuum circuit breaker online; based on the analysis of on-line monitoring requirements of medium voltage vacuum circuit breaker, a general design framework of on-line monitoring system for medium voltage vacuum circuit breaker is designed in reference [6], and several key technical problems in the process of software and hardware design that need to be solved in the process of on-line monitoring of circuit breaker are introduced in detail. The on-line monitoring system developed according to the technical scheme in this paper has been applied to many products Successful application, perfect function, good performance and high reliability. But the accuracy of the above two methods is low.

In view of the above problems, this paper proposes an embedded intelligent vacuum monitoring method based on the Internet of things.

\section{Internet of things technology}

The Internet of things refers to the real-time collection of any object or process that needs to be monitored, connected and interacted through sensors, RFID technology, global positioning system and other technologies, as well as the collection of all kinds of required information such as sound, light, heat, electricity, mechanics, chemistry, biology, location, etc. through various possible network access, it can realize the ubiquitous link between things and things, things and people, and realize the connection between objects and people Intelligent perception, recognition and management of process. Internet of things technology is an extension and extension of Internet technology. Through Internet of things technology, the equipment is automatically and real-time identified, located, tracked, monitored and triggered corresponding events. It is important to carry 
out wireless transmission of operation data and monitoring instructions to achieve real-time management and control of the equipment. By using the Internet of things technology, through the perception of the outside world, an intelligent vacuum monitoring network of high-voltage circuit breakers is constructed to comprehensively monitor the factors affecting the operation of high-voltage circuit breakers [7-9]. The application of RFID, ZigBee and other Internet of things technology to the design of high-voltage circuit breaker not only makes it have the function of traditional high-voltage circuit breaker, but also realizes identification, monitoring and control. Finally, the integrated monitoring host follows IEC 61850 protocol and transmits data to the integrated information platform through optical fiber. Its main technical features are: low power consumption, large network capacity, short time delay, reliability, security and so on.

\section{Monitoring principle}

The embedded intelligent high-voltage circuit breaker relies on the vacuum in the arc extinguishing chamber to complete the arc process. The theory of gas discharge shows that whether the collision ionization and recombination of charged particles in gas are balanced determines whether the gas decomposes due to the discharge phenomenon or has the electrical strength characteristics of insulation. In the environment of constant temperature, electric field and polar distance, vacuum degree is the main reason of gas molecular movement in the arc extinguishing chamber. The larger the free path of charged particles is, the more difficult it is to collide in the process of motion. In high vacuum environment, the collision ionization between charged particles is weakened. At this time, the collision ionization and recombination of charged particles are in equilibrium state [10-12]. The structure of vacuum interrupter is shown in Figure 1.

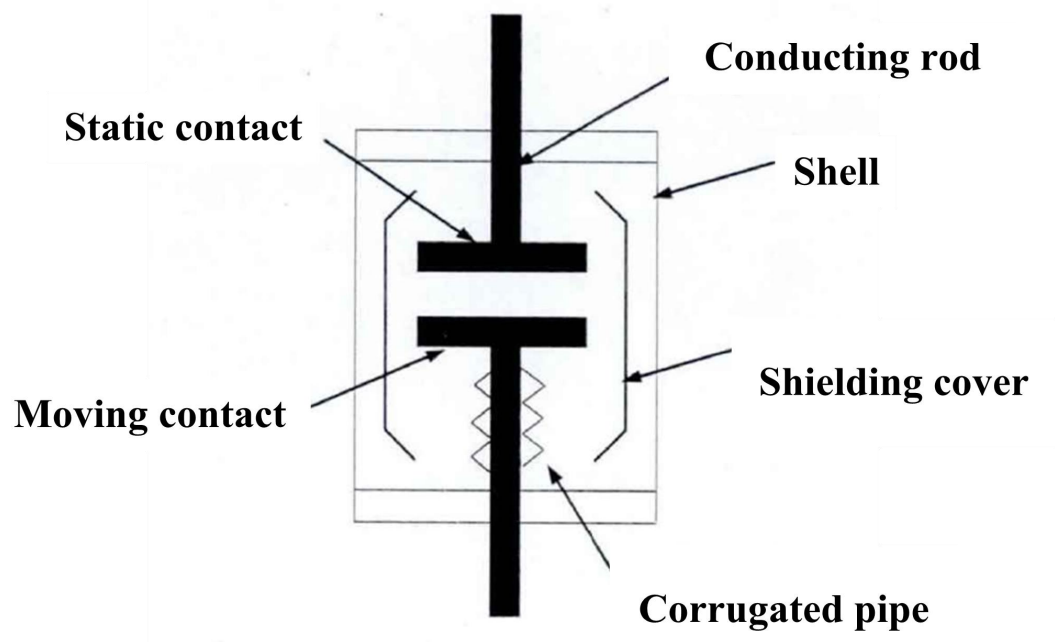

Fig. 1. Vacuum interrupter structure 
When the vacuum degree in the arc extinguishing chamber is high enough, only a small voltage value is needed to maintain the electron flow generated by field emission between the contact and the shield. At this time, the free path of the electron is smaller than the distance between the electrode and the shield. The electron reaches the shield without collision, and the potential on the shield increases as the charge accumulates on the shield. The negative charge accumulated on the shield will make it the negative potential reaches a peak [13-14]. When the vacuum inside the shield deteriorates, the pressure of the gas becomes larger and the particles such as gas molecules become more, which leads to the decrease of the insulation characteristics of the gas and the pre breakdown voltage. It is easy to have pre breakdown and pre discharge, and the potential on the shield will decrease. When the real void is close to the critical value, the travel of electrons will be smaller, and fierce collision will occur before reaching the enclosure wall. The internal gas molecules will absorb the electrons produced by field emission and form heavy and slow drifting negative ions. There is almost no charge accumulation on the enclosure wall [15-16]. The above analysis shows that the potential change on the shield can reflect the internal vacuum of the embedded intelligent high voltage circuit breaker. Figure 2 shows the change curve of AC potential and DC potential of shield.

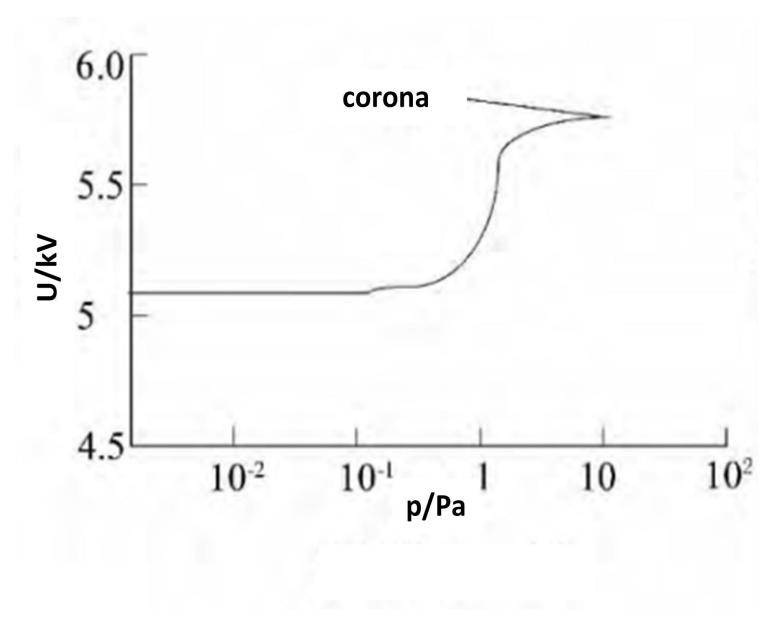

(a)AC potential 


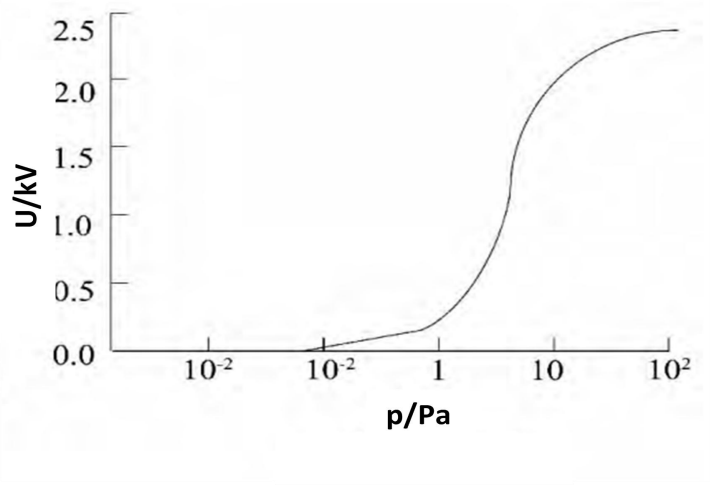

(b)DC potential

Fig. 2. Change curve of AC potential and DC potential of shield

At present, there are many on-line monitoring methods for the vacuum degree of embedded intelligent high-voltage circuit breaker, among which the coupling capacitance method is widely used. The coupling capacitance method is developed on the basis of the potential change detection method. According to the change of the surrounding electric field caused by the change of the potential on the shield, the change of the potential measured by the coupling voltage probe outside the shield wall is used to indirectly obtain the change of the gas pressure inside the arc extinguishing chamber [17]. The main idea is to insert a detection contact between the vacuum bubble cover and the earth, and keep a certain distance between the contact and the vacuum bubble cover, so that the detection contact will not affect the safe and stable operation of the circuit breaker. The distance between the detection contact and the vacuum bubble shield can be calculated according to the theory or the test. The schematic diagram is shown in Figure 3.

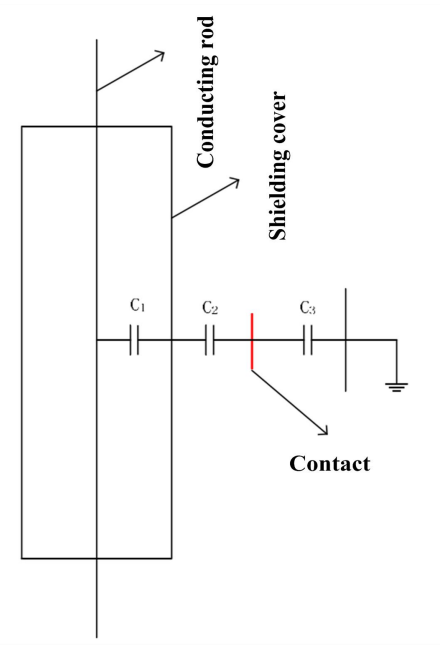


Fig. 3. Equivalent capacitance diagram of coupling capacitance method

In Figure 3, C1 is the capacitance between the conducting rod and the shield. The capacitance value has been determined when the switch leaves the factory, and it is very stable as long as the vacuum degree of the arc extinguishing chamber remains unchanged; $\mathrm{C} 2$ is the capacitance between the shield and the contact. The capacitance value is basically determined by the area of the contact and the distance between the contact and the shield. In fact, as long as the position of the detection contact is fixed, the capacitance value remains unchanged; C3 is the capacitance value between the contact and the earth. Like $\mathrm{C} 2$, the capacitance value is basically determined by the area of the contact and the distance between the contact and the shield. In fact, as long as the position of the detection contact is fixed, the capacitance value remains unchanged. In practice, the capacitance of $\mathrm{C} 1$ and $\mathrm{C} 2$ is much larger than that of $\mathrm{C} 3$. In order to increase the capacitance of $\mathrm{C} 2$ as much as possible, solid materials with large dielectric constant are filled between the contact and the shield. In normal operation, three equivalent capacitors are connected in series to divide the voltage. Since the capacitance value of $\mathrm{C} 2$ is the largest, the voltage of $\mathrm{C} 2$ is the smallest. As long as the air pressure of vacuum bubble changes slightly, the relative value of the voltage change on $\mathrm{C} 2$ will be large, which greatly improves the sensitivity of monitoring. In addition, the capacitance value of $\mathrm{C} 2$ can be adjusted according to the actual needs, which greatly facilitates the design of online monitoring system. In fact, $\mathrm{C} 2$ can be designed as adjustable capacitance to cooperate with the commissioning and sensitivity test of online monitoring system [18-20].

\section{Embedded intelligent vacuum monitoring of high voltage circuit breaker based on Internet of things}

According to the monitoring principle, a low-cost and low-power short-distance wireless network communication technology is applied to the vacuum degree monitoring of embedded intelligent high-voltage circuit breaker. Through online monitoring of the vacuum degree parameters of embedded intelligent high-voltage circuit breaker, the fault analysis of high-voltage circuit breaker is realized [21-22]. The CC2530 chip produced by TI company is selected for wireless communication control chip, which is based on the wireless technology of pressure ee802.15.4 protocol. The chip of ZigBee technology integrates the enhanced 8051 single-chip microcomputer core, which can directly write programs. The chip model used is cc2530f 256 . The structure size is small, the development board size is reduced, the cost is reduced, and the receiving signal is sensitive, With strong anti-interference ability and few external components, the direct communication distance between hardware nodes can reach more than 100 meters by using a suitable and simple main circuit [23-25].

The Internet of things needs to continuously sense the vacuum degree data of high-voltage circuit breaker according to multiple sensor nodes, because the vacuum degree data of 
high-voltage circuit breaker received by sensor nodes has the disadvantages of complex, variable, numerous and poor repeatability, which has great redundancy in the time and space of vacuum degree data detection of high-voltage circuit breaker. Therefore, based on the analysis of the network boundary effect, using the Internet of things technology, the vacuum data flow of high-voltage circuit breakers can fundamentally reduce the redundant vacuum data of high-voltage circuit breakers, and reduce the energy consumption of sensor nodes. In this paper, the short-range wireless network communication technology, according to the basic parameter replication mechanism, can fundamentally reduce the generation of redundant high-voltage circuit breaker vacuum data, and can more effectively reduce the errors in the calculation process.

If a node in a cluster in this sensor network is $S_{0}, S_{1}, \ldots, S_{n}$, then the sequence instruction received by the $y$ node is $C_{y, c o l}$, and the basic form of the sequence is $C_{y, d o l}=\left(C_{0, y}+C_{1, y}+\ldots+C_{n-1, y}\right)^{T}$. in the sequence matrix, each sensor node can identify $n$ high-voltage circuit breaker vacuum data points, in which $C_{j, y}$ represents the high-voltage circuit breaker vacuum data in a node. According to all sensor nodes containing the vacuum degree data of high-voltage circuit breaker, the basic sequence matrix $n \times N$ of vacuum degree data $C^{0}$ of high-voltage circuit breaker can be constructed:

$$
\begin{aligned}
C^{0} & =\left(C_{0 c o l}+C_{1 c o l}+\ldots+C_{(n-1) c o l}\right) \\
& =\left\{\begin{array}{ccccc}
C_{0,0} & C_{0,1} & \ldots & C_{0, n-1} \\
C_{1,0} & C_{1,1} & \ldots & C_{1, n-1} \\
\vdots & \vdots & \vdots \\
C_{N, 0} & C_{N, 1} & \ldots & C_{N, n}
\end{array}\right.
\end{aligned}
$$

Among them, the matrix presents the instructions received by any sensor node, specifically the vacuum data flow of the $n$-th high-voltage circuit breaker over time; the row of the matrix

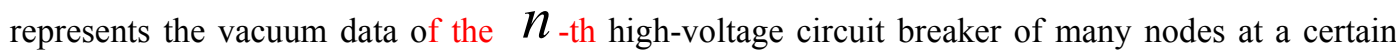
time. On this basis, the column and row transformation for equation (1) refers to the transformation of time $S$ and space $d$, and the transformation formula is as follows:

$$
C_{1}{ }^{0}=\left\{\begin{array}{cccc}
S^{1}{ }_{0,0} & S^{1}{ }_{0,1} & \ldots & S^{1}{ }_{0, n-1} \\
\vdots & \vdots & & \vdots \\
S_{N-1,0}^{1} & S^{1}{ }_{N-1,1} & \ldots & S^{1}{ }_{N-1, n-1} \\
d^{1}{ }_{0,0} & d^{1}{ }_{0,1} & \cdots & d^{1}{ }_{0, n-1} \\
\vdots & \vdots & & \vdots \\
d_{N-1,0}^{1} & d_{N-1,1}^{1} & \ldots & d_{N-1, n-1}^{1}
\end{array}\right.
$$


Where, $C_{1}^{0}$ represents the vacuum degree data of the transformed high-voltage circuit breaker. The algorithm monitors the data transmission time point, because there will be a lot of data reduction in the process of data transformation, so this step is also to reduce the energy consumption of node transmission in the sequence matrix row transformation. The row transformation formula is:

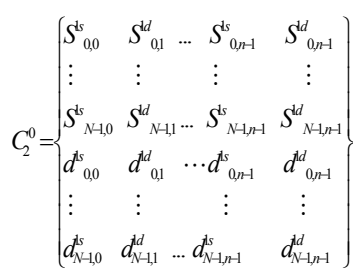

Where, $C_{2}^{0}$ refers to the vacuum degree data of high-voltage circuit breaker after row transformation. Compared with the data flow of vacuum degree of high-voltage circuit breaker initially received by nodes, the amount of redundant vacuum degree data of high-voltage circuit breaker is much less, and more free time space is also released. After transformation, according to formula (3), we can further get:

$$
C^{1}=\left\{\begin{array}{cccc}
S_{0,0}^{1 s} & S^{1 s} & \cdots & S_{0, n-1}^{1 s} \\
S^{1 s} & S_{1,1}^{1 s} & \cdots & S_{1,1, n-1}^{1 s} \\
\vdots & \vdots & & \vdots \\
S_{N-1,0}^{1 s} & S_{N-1,1}^{1 d} & \cdots & S_{N-1, n-1}^{1 d}
\end{array}\right\}
$$

In the formula, $C^{1}$ represents the vacuum degree data of high-voltage circuit breaker after time and space release. According to the same way above, the data of vacuum degree of high-voltage circuit breaker is transformed. On the basis of the data change of vacuum degree of high-voltage circuit breaker, the corresponding recursive processing is done, and the wavelet coefficients of different degrees are obtained. Finally, the wavelet coefficient is compared with the original vacuum degree data of high-voltage circuit breaker. According to the comparison results, the transformed vacuum degree data of high-voltage circuit breaker greatly reduces the pressure of time and space to a certain extent, which is very important for the current energy limited Internet of things.

According to the wavelet function, a distributed transform is designed, which is suitable for any transform support length. By removing the spatial-temporal correlation of the vacuum degree data of the high-voltage circuit breaker in each node of the ring, the redundant phenomenon of the vacuum degree data of the high-voltage circuit breaker can be reduced to a certain extent, and then the transmission efficiency of the vacuum degree data of the high-voltage circuit breaker between each node of the ring and the cluster head node can be realized. 
Considering that any cluster in the Internet of things uses $N$ node: $S_{0}, S_{1}, \ldots, S_{i}, \ldots, S_{N-1}$ to construct a ring, assuming that the vacuum degree data of the high-voltage circuit breaker stored in the $y$ node $S_{y}$ of the ring is represented by $c_{y}$, then the vacuum degree data of the high-voltage circuit breaker collected by this cluster can be represented as a whole vector set: $d=\left(c_{0}, c_{1}, \ldots, c_{N-1}\right)$. This vector set needs to pay attention to the difference between $d$ and other ordinary vectors. In the ring model, the 0 th element $c_{0}$ of vector $d$ is fundamentally adjacent to the last element $c_{N-1}$.

The adjacent nodes in the ring belong to the virtual network adjacent to each other in space, so the vacuum degree data of high-voltage circuit breaker stored in the adjacent nodes in the ring may have higher spatial correlation. On the basis of the distribution transformation of the ring model, the calculation of each low-frequency or high-frequency coefficient is aimed at an adjacent node of the support length, so the spatial correlation can be used to eliminate the redundant vacuum data.

The effective way to solve the boundary effect is to expand the boundary, and link the sensor nodes into a ring, which is equivalent to the periodic expansion of the signal, so as to solve the problem of boundary effect, and realize the efficient monitoring of vacuum degree data of high-voltage circuit breaker.

\section{Simulation experiment}

In order to verify the validity of the embedded intelligent vacuum monitoring method based on the Internet of things, a simulation experiment is carried out. The experimental environment is shown in Table 1.

Table 1 Configuration information of development environment

\begin{tabular}{cc} 
Table 1 Configuration information of development environment \\
\hline Name & Configuration \\
\hline Operating system & Microsoft Windows XP \\
Processor & Intel(R)Celeron(R) $2.6 \mathrm{GHz}$ \\
Internal storage & $6.0 \mathrm{~GB}$ \\
\hline
\end{tabular}


Hard disk

Database management software

JDK
$4.0 \mathrm{~GB}$

Microsoft SQL server 2010 R2

1.6

Mathematical software

MATLAB

Take the high-voltage circuit breaker of $126 \mathrm{kV}$ electronic equipment as an example, monitor the vacuum degree of the high-voltage circuit breaker, and build the experimental platform as shown in Figure 4.

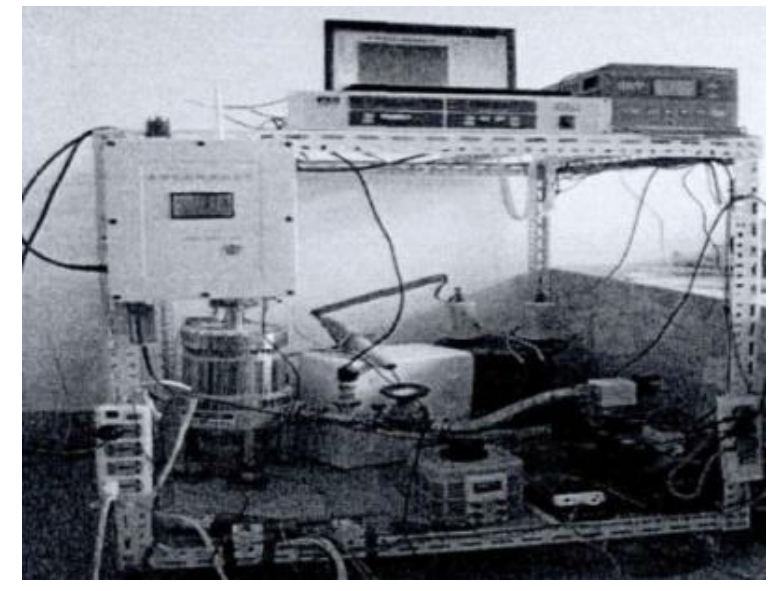

Fig. 4. Construction of Experimental Platform

Set parameters of high-voltage circuit breaker in the experimental platform, as shown in Table 2.

Table 2 Parameters of high voltage circuit breaker

\begin{tabular}{cccc}
\hline Order number & Name & Unit & Numerical value \\
\hline 1 & $\begin{array}{c}\text { Rated voltage } \\
\text { Power }\end{array}$ & $\mathrm{kV}$ & 12 \\
& frequency & & \\
withstand & $\mathrm{kV}$ & 42 \\
voltage & & \\
Rated & & 75 \\
& lightning & $\mathrm{kV}$ & \\
\hline
\end{tabular}




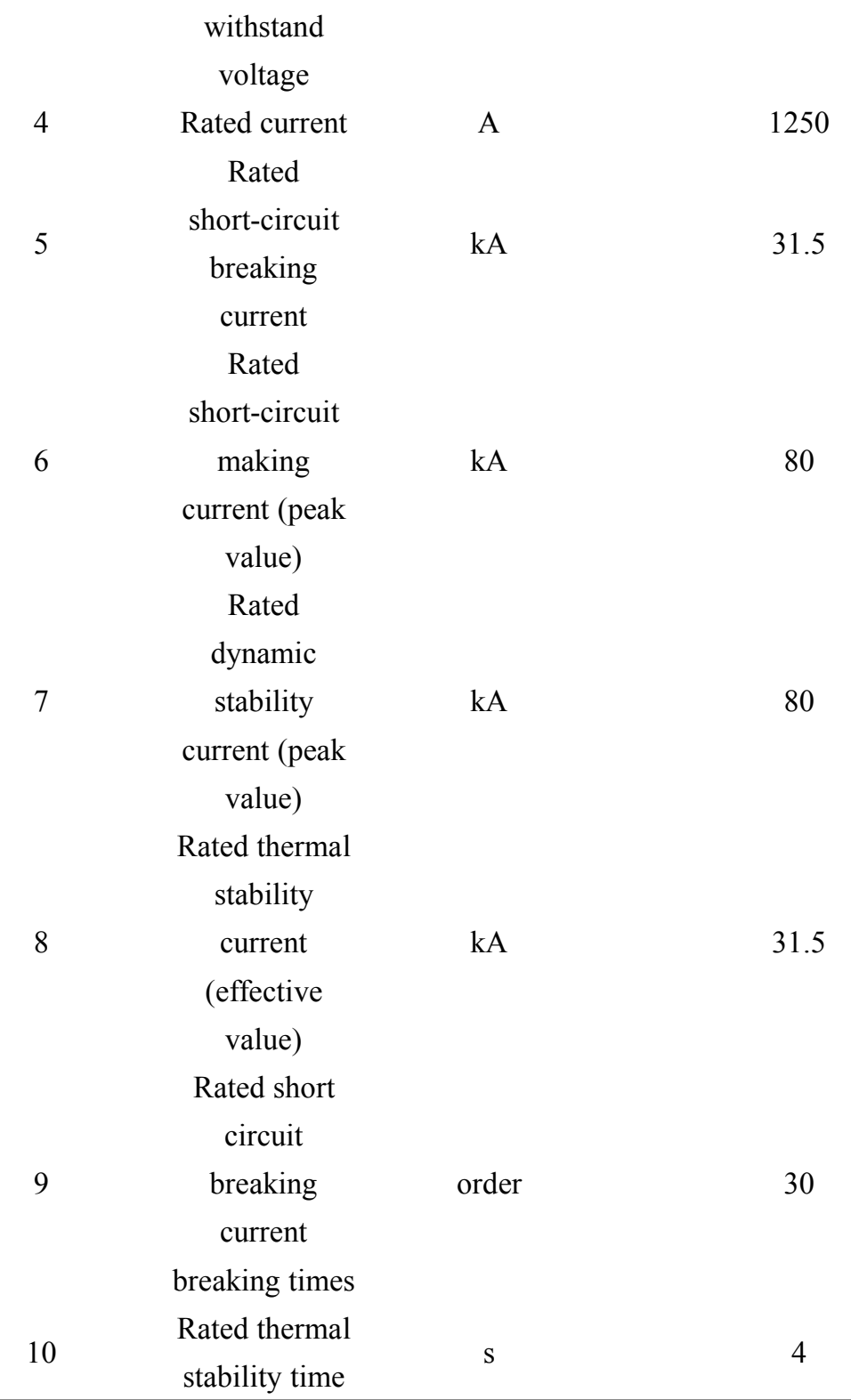

According to the parameters of the high-voltage circuit breaker, through the vacuum condition in the arc extinguishing chamber of the high-voltage circuit breaker, and by changing the voltage between the breaks of the circuit breaker, the change curve between the breakdown voltage between the breaks and the vacuum degree in the arc extinguishing chamber is obtained, as shown in Figure 5. 


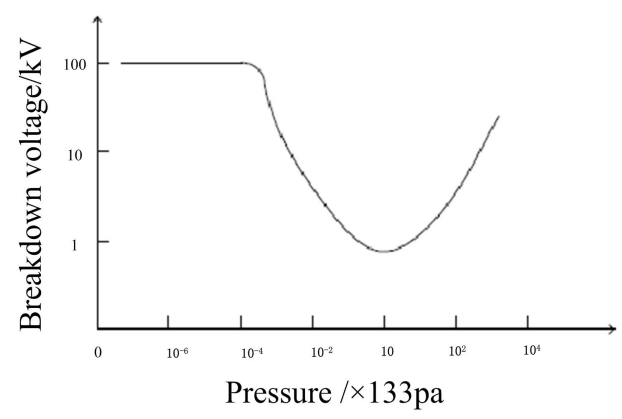

Fig. 5. Curve of breakdown voltage of circuit breaker with pressure in arc extinguishing chamber

It can be seen from the figure that when the pressure in the arc extinguishing chamber is less than $1.33 \times 10-2 \mathrm{~Pa}$, the withstand voltage strength of the arc extinguishing chamber is high and almost unchanged, about $100 \mathrm{kV}$. When the pressure in the arc extinguishing chamber is greater than $0.133 \mathrm{~Pa}$, the breakdown voltage of the fracture gradually decreases, and when the voltage is about $0.93 \mathrm{pa}$, the breakdown voltage of the fracture reaches the minimum value, less than $1 \mathrm{kV}$, and then increases with the pressure, The breakdown voltage between ports increases gradually. It shows that this method can effectively monitor the vacuum degree of the embedded intelligent high voltage circuit breaker.

In order to further verify the effectiveness of this method, the monitoring accuracy of this method, literature [5] method and literature [6] method are compared and analyzed, and the comparison results are shown in Figure 6.

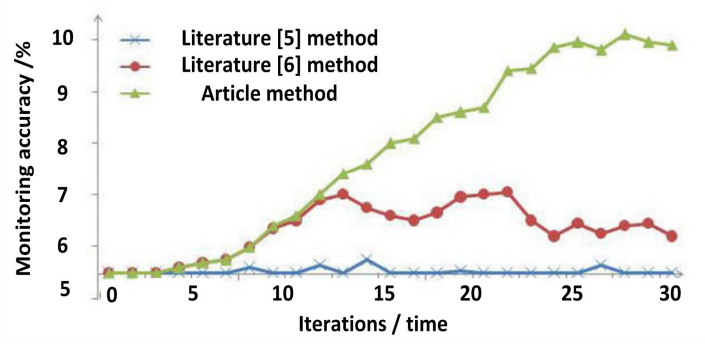

Fig. 6. Comparison results of monitoring accuracy

According to figure 6, the monitoring accuracy of the embedded intelligent high-voltage circuit breaker vacuum degree in this method can reach up to $100 \%$, which is higher than that of the literature method.

\section{Concluding remarks}

Due to the low accuracy of the traditional embedded intelligent high-voltage circuit breaker vacuum monitoring method, this paper proposes an embedded intelligent high-voltage circuit 
breaker vacuum monitoring method based on the Internet of things. Based on the principle of embedded intelligent high-voltage circuit breaker vacuum degree monitoring and the Internet of things sensing technology, the embedded intelligent high-voltage circuit breaker vacuum degree is monitored. The experimental results show that this method can accurately monitor the vacuum degree of intelligent high-voltage circuit breaker and promote the construction and development of intelligent power grid.

\section{Fund projects}

Major Project of the Ministry of Education: "Research on the Construction of Ethnic Disciplines and Majors in Ethnic Areas: Cases of Inner Mongolia and Northeast Ethnic Universities" (number: mjzxzd1405)

\section{References}

[1] S. M. Ghafourian, I. Arana, J. Holbøll, et al. General Analysis of Vacuum Circuit Breaker Switching Overvoltages in Offshore Wind Farms. IEEE Transactions on Power Delivery, Vol. 31, no. 5, pp. 2351-2359 (2016)

[2] C.L. Bak, A. Borghetti, J. Glasdam, et al. Vacuum circuit breaker modelling for the assessment of transient recovery voltages: Application to various network configurations. Electric Power Systems Research, Vol. 156, pp. 35-43 (2018)

[3] J. Liu, W. Shi, P. Li. Analysis on Two Applications of Fast Vacuum Circuit Breaker. High Voltage Apparatus, Vol. 53, no. 3, pp. 185-189 (2017)

[4] Hou C, Yu X, Cao Y, et al. Prediction of synchronous closing time of permanent magnetic actuator for vacuum circuit breaker based on PSO-BP. IEEE Transactions on Dielectrics \& Electrical Insulation, Vol. 24, no. 6, pp. 3321-3326 (2018)

[5] Yuan Xiaonan, He Wenlin. On-line Testing System for Vacuum Degree of High Voltage Vacuum Circuit Breaker . Electrical electrician, no. 6, pp. $54-57$ (2018)

[6] Xu Jianguo, Chen Haikun, Dai Yunxia, et al. Research on on-line monitoring system of vacuum circuit breaker . Electronic Technology and Software Engineering, no. 9, pp. 201-204 (2016)

[7] W. Zhou, X. Liu, C. Huang, et al. Optimization and Simulation of Commutation Circuit of $60 \mathrm{kV}$ Double-Break Vacuum Circuit Breaker. Zhenkong Kexue Yu Jishu Xuebao/journal of Vacuum Science \& Technology, Vol. 37, no. 9, pp. 885-889 (2017)

[8] Jiaming Jiang, Heyun Lin, Shuhua Fang. Optimization Design of a Permanent Magnet Actuator for 126 kV Vacuum Circuit Breaker. IEEE Transactions on Applied Superconductivity, no. 99, pp. 1-1 (2017)

[9] Q. Peng, X. Jiang, W. Sima, et al. Three-phase Modeling of the Vacuum Circuit Breaker Switching off 35 kV Shunt Reactors and Overvoltage Suppression. Gaoya Dianqi/high Voltage Apparatus, Vol. 53, no. 3, pp. 147-159 (2017)

[10]Dullni E, Lindell E, Liljestrand L. Dependence of the Chopping Current Level of a Vacuum Interrupter on 
Parallel Capacitance., no. 99, pp. 1-7 (2017)

[11]Z. Shi, J. Sun, F. Pan, et al. Overvoltage Simulation and Protection of $126 \mathrm{kV}$ Vacuum Circuit Breaker. High Voltage Apparatus, Vol. 53, no. 3, pp. 132-140 (2017)

[12]F. Guo, X. Liu, G. Zhu, et al. Characteristic analysis of permanent magnet linear motor operating mechanism for $12 \mathrm{kV}$ vacuum circuit breaker. Power System Protection \& Control, Vol. 46, no. 1, pp. 37-43 (2018)

[13]Zhai Xiaoshe, Tian Limin, Lan Zhenbo, et al. Experimental Research on Optimal Arcing Time of $40.5 \mathrm{kV}$ Double-break Vacuum Circuit Breaker During Capacitive Interruption Process. High Voltage Apparatus, Vol. 53, no. 3, pp. 160-166 (2017)

[14]P. Widger, A. Haddad, H. Griffiths. Breakdown performance of vacuum circuit breakers using alternative CF 3 I-CO 2 insulation gas mixture. IEEE Transactions on Dielectrics \& Electrical Insulation, Vol. 23, no. 1, pp. 14-21 (2016)

[15]LI Yi, JIANG Hao, GONG Fugao, et al. Online Monitoring System of Intelligent Circuit Breaker Based on Virtual Instrument Technology. High Voltage Apparatus, Vol. 53, no. 6, pp. 67-71 (2017)

[16]X. Cheng, X. Zhu, Y. Du, et al. High Voltage Circuit Breaker Fault Diagnosis Based on Neural Fuzzy Petri Nets. Transactions of China Electrotechnical Society, Vol. 33, no. 11, pp. 2535-2544 (2018)

[17]L. Zhao, R. Fu, Q. Rong, et al. State Evaluation of High Voltage Circuit Breaker Operating Mechanism Based on Adaptive Neural Fuzzy Inference System. Gaodianya Jishu/high Voltage Engineering, Vol. 43, no. 6, pp. 2007-2015 (2017)

[18]R. Fu, L. Zhao, Q. Rong, et al. Study on Mechanical Characteristics of Operating Mechanism of High Voltage Circuit Breaker. High Voltage Apparatus, Vol. 53, no. 5, pp. 56-62 (2017)

[19]G. Tian, C. Wang, Z. Wang, et al. Thermal Characteristics Analysis of High Voltage Tank Type Circuit Breaker. High Voltage Apparatus, Vol. 53, no. 6, pp. 141-146 (2017)

[20]WU Xueguang, WANG Xiaochen, ZHU Yongqiang, et al. Breaking and Simulation Method of Arrestors Step-by-step Operating of High Voltage DC Circuit Breaker. High Voltage Engineering, Vol. 43, no. 4, pp. 1079-1085 (2017)

[21]L. Wang, L. Jing, D. Xu, et al. Improvement and Simulation of the Motor Actuator Rotor for High Voltage Vacuum Circuit Breaker. Gaoya Dianqi/high Voltage Apparatus, Vol. 53, no. 2, pp. 61-67 (2017)

[22]Xiaoqing Song, Chang Peng, Alex Q. Huang. A Medium-Voltage Hybrid DC Circuit Breaker, Part I: Solid-State Main Breaker Based on 15 kV SiC Emitter Turn-OFF Thyristor. IEEE Journal of Emerging \& Selected Topics in Power Electronics, Vol. 5, no. 1, pp. 278-288 (2017)

[23]Li Xingwen, Qu Jianyu, Wang Qian. Influences of Arc Plate on Arc-quenching Performance of Bridge-shape Double-break Circuit Breaker. High Voltage Engineering, Vol. 43, no. 3, pp. 845-850 (2017)

[24]Q. Liu, L. Wang, P. Liu. Measurement of Radiation Electric Field Produced by Action of GIS Circuit Breaker. Gaoya Dianqi/high Voltage Apparatus, Vol. 53, no. 2, pp. 166-171 (2017) 
[25]WANG Chengiia, TANG Li, LI Xinwei, et al. Effect of Metallic Particles on Electric Field of AC Filter Circuit Breaker Arc-extinguishing Chamber. High Voltage Apparatus, Vol. 53, no. 6, pp. 105-112 (2017) 